\title{
Tailor-made PL-UC-C3 nanoparticles for fluorescence/computed tomography imaging- guided cascade amplified photothermal therapy
}

This article was published in the following Dove Press journal: International Journal of Nanomedicine

\author{
Xinhui Xie' \\ Jialei Song' \\ Yili $\mathrm{Hu}^{\prime}$ \\ Suyang Zhuang' \\ Yuntao Wang' \\ Yunlei Zhao ${ }^{2}$ \\ Qian Lu
}

'Department of Orthopedics, Zhong Da Hospital, School of Medicine, Southeast University, Nanjing, Jiangsu 210093, China; ${ }^{2}$ Department of Biomedical Engineering, College of Engineering and Applied Sciences, Nanjing University, Nanjing, Jiangsu 210093, China
Correspondence: Xinhui Xie Department of Orthopedics, Zhong Da Hospital, School of Medicine, Southeast University, 87 Dingjiaqiao Road, Nanjing, Jiangsu 210093 , China

Tel +86 I395 I99 950 I

Email xiexinghuixxh@163.com

Qian Lu

Department of Biomedical Engineering, College of Engineering and Applied Sciences, Nanjing University, 22 Hankou Road, Nanjing, Jiangsu 210093, China Tel +86 I58 50672529

Email qianlu@nju.edu.cn
Background: Development of the burgeoning number of photothermal therapy (PTT) agents has drawn a huge amount of interest, since PTT treatment is a powerful and effective alternative to traditional treatments. Optimal PTT agents should integrate some essential preconditions including negligible systemic toxicity, deep penetration into tumor tissues, and maximum laser energy absorbance. Unfortunately, only few of the PTT agents reported could meet all of the above mentioned conditions.

Methods: Here, we report a brand new PTT agent through the encapsulation of $\mathrm{NaGdF}_{4}: \mathrm{Yb}, \mathrm{Tm} @$ $\mathrm{NaGdF}_{4}: \mathrm{Yb}$ (UCNPs) and an organic compound (C3) into poly-e-caprolactone-polyethylenepolyglycol (PCL-PEG) (PL-UC-C3 NPs).

Results: UCNPs as an up-conversion material and C3 as a PTT agent both feature low cytotoxicity, and most importantly, UCNPs with superior conversion efficiency could efficiently absorb the energy of a $980 \mathrm{~nm}$ laser, transform the near-infrared laser light into visible light, and translate the palingenetic visible light to $\mathrm{C} 3$. The usage of a $980 \mathrm{~nm}$ laser ensures a deeper penetration and lower energy, while the highly efficient absorption and transformation process confers a cascade amplified hyperthermia for tumor treatment.

Conclusion: In this regard, our research provides a powerful and robust breakthrough for florescence/computed tomography imaging-guided PTT treatment, lighting up the clinical application in cancer treatment.

Keywords: photothermal therapy, PCL-PEG, up-conversion, excellent photothermal stability, high heat conversion efficiency

\section{Introduction}

Despite the 5-year survival rate being modestly elevated, mainly owing to the advances in traditional cancer treatments including chemotherapy, radiotherapy, and surgery, most cancer patients cannot be completely cured and continue to suffer from tumor recurrence and tumor metastasis. Two leading causes can be accounted for this condition. On the one hand, the tumor treatment is impressively blocked by the tumor microenvironment. ${ }^{1,2}$ The extreme conditions existing in tumor microenvironment, such as hypoxia, metabolic stress, insufficient blood supply, and so on, endow tumor cells with treatment resistance and the ability to survive adversity. ${ }^{3,4}$ On the other hand, traditional cancer treatments face dire challenges due to chemotherapeutic tolerance in chemotherapy, side effect in radiotherapy, and invisible small lesions in surgery. ${ }^{5,6}$ As such, seeking new avenues for tumor treatment is of paramount significance in clinical application. 


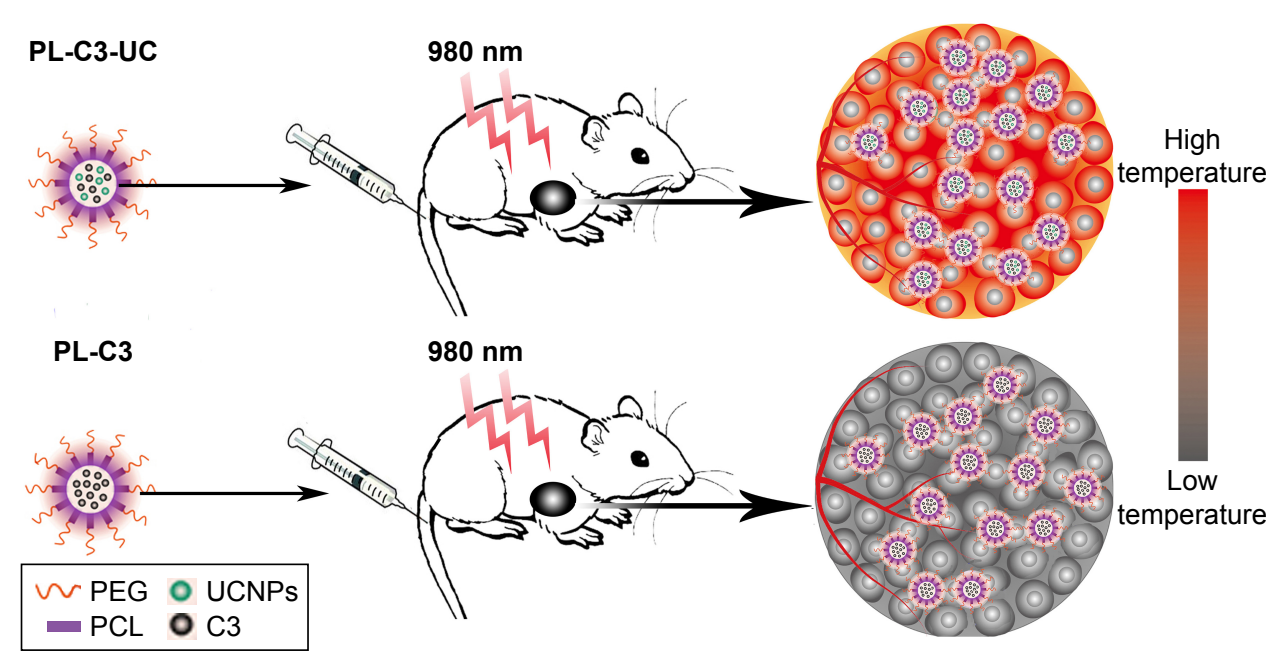

Figure I When the PL-C3-UC or PL-C3 nanoparticles were injected into the body, they were stable and had prolonged circulation life in the blood. Notes: After they reached the tumor area via the EPR effect, a $980 \mathrm{~nm}$ laser was employed to irradiate the lesion to obtain a local high temperature. In PL-C3-UC group, UCNPs with superior conversion efficiency could efficiently absorb the energy of the $980 \mathrm{~nm}$ laser, transform the NIR laser light into visible light, and translate the palingenetic visible light to $\mathrm{C} 3$, thereby robustly conferring a cascade amplified hyperthermia for tumor treatment. C3, organic compound; PL-C3-UC, encapsulation of UCNPs and C3 into PEG-PCL; PL-C3, encapsulation of C3 into PEG-PCL.

Abbreviations: PEG, polyethylene-polyglycol; PCL, poly-e-caprolactone; UCNPs, up-conversion nanoparticles; EPR, enhanced permeability and retention effect; NIR, near infrared.

Photothermal therapy (PTT), characterized by the selective transformation of absorbed light into heat, has been increasingly regarded as a potential alternative to traditional cancer treatments attributed to the noninvasive nature and highly effective tumor killing. ${ }^{7}$ To prevent the surrounding tissues from being affected in the process of tumor hyperthermia ablation, ideal photothermal agents which are capable of absorbing near-infrared (NIR) laser and converting the absorbed laser into heat are urgently desired to be designed. ${ }^{8-16}$ Recently, various PTT agents have been investigated, including noblemetal nanomaterials, polymer nanoparticles, semiconductor nanomaterials, and carbon-based nanomaterials, because they exhibit strong NIR absorbency. ${ }^{17-24}$ It is successful to some extent because NIR laser-induced PTT is bound to avoid the thermal ablation of normal tissue owing to deep penetration and low energy of NIR laser irradiation. However, most PTT agents, especially metal-based PTT agents, feature high toxicity to normal tissues, therefore restricting their clinical application. ${ }^{25}$ Research community has been attempting to leverage organic nanomaterials which have low toxicity as alternatives, and relevant studies have been recently reported. ${ }^{26,27}$

Previously, we tailor-made an organic nanomaterial named $\mathrm{C} 3$, which was used to eradicate tumor by combination with another photodynamic therapy (PDT) agent named indocyanine green (ICG). ${ }^{18}$ In that study, without the help of PDT, PTT alone rendered by $\mathrm{C} 3$ under $808 \mathrm{~nm}$ laser irradiation played a small role in tumor inhibition. It is reasonable that $\mathrm{C} 3$ has poor absorbency in NIR. Note that $\mathrm{C} 3$ has an extremely high absorption peak at $450 \mathrm{~nm}$, thus visible light maybe more appropriate to be utilized in this platform. However, visible light suffers from a shallower penetration, thus wasting the laser energy and offering ineffective hyperthermia for tumor treatment.

It was reported that gadolinium-based up-conversion nanoparticles (Gd UCNPs) were extensively utilized to arm the phototherapy and the conversion efficiency of Gd UCNPs increased in the wake of increasing the mix proportion of ytterbium $(\mathrm{Yb})$ to some extent. ${ }^{28}$ Based on this, we prepared PL-UC-C3 NPs, in which UCNPs with superior conversion efficiency were employed to absorb NIR laser light and transform it into visible light. In this platform, both $\mathrm{C} 3$ and UCNPs feature low cytotoxicity as demonstrated via MTT assay. Taking advantage of a $980 \mathrm{~nm}$ laser, deep penetration and low energy were acquired, thus causing little damage to normal tissues. After the transformation of NIR laser light into visible light and the subsequent capture of visible light by $\mathrm{C} 3$, an impressive hyperthermia was generated as analyzed by the in vivo data. As a proof-of-concept study, the feasibility of our platform to cascade convert NIR laser light into visible light achieves the highly effective tumor inhibition and the less damage to normal tissues. The whole process is illustrated in Figure 1. Collectively, our research provides a powerful and robust platform for the cascade conversion in PTT, providing a promising protocol for clinical application in cancer treatment.

\section{Materials and methods Materials and animals}

Thulium oxide $\left(\operatorname{Tm}_{2} \mathrm{O}_{3}, 99.9 \%\right)$, gadolinium oxide $\left(\mathrm{Gd}_{2} \mathrm{O}_{3}\right.$, 99.9\%), and ytterbium oxide $\left(\mathrm{Yb}_{2} \mathrm{O}_{3}, 99.9 \%\right)$ were supplied from Linen Technology Development Co. Ltd. (Shanghai, 
China). 1-Octadecene (ODE, $>90 \%$ ), oleylamine $(>80 \%)$, oleic acid $(\mathrm{OA},>90 \%)$, trifluoroacetic acid sodium salt ( $\left.\mathrm{NaCF}_{3} \mathrm{COO}, 99 \%\right)$, trifluoroacetic acid $\left(\mathrm{CF}_{3} \mathrm{COOH}, 99 \%\right)$, poly-e-caprolactone-polyethylene (PCL, 98\%), and polyglycol (PEG, 98\%) were acquired from J\&K Science (Beijing, China). Ethanol (AR) and chloral hydrate (AR) were obtained from Shanghai Lingfeng Chemical Reagent Co., Ltd. (Shanghai, China). MTT, FBS, DMEM, buffered saline (PBS, pH =7.4), trypsin, LysoTracker Red DND-99 Kit, and Annexin V-FITC Apoptosis Staining/Detection Kit were purchased from KeyGEN BioTECH (Nanjing, China). All chemicals in this experiment were used as received without further purification. Both the human umbilical vein endothelial cells (HUVECs) and murine mammary carcinoma cell lines (4T1) were obtained from the Nanjing Institute of Cell Biology (Nanjing, China). All cell experiment protocols, methods, and procedures were performed in accordance with relevant guidelines and regulations of the Medical Ethics Review Committee of Nanjing University. The Balb/c mice (female, 4-6 weeks) were bought from Comparative Medicine Center of Yangzhou University. Animal experiments were approved by the Care Committee of Nanjing University (including guidelines for animal care and use, and guidelines for euthanasia of mice, Protocol \#: 20170411-015).

\section{Synthesis of $\operatorname{Ln}\left(\mathrm{CF}_{3} \mathrm{COO}\right)_{3}$}

An appropriate amount of lanthanide oxide was mixed with excessive deionized water and trifluoroacetic acid, and then the mixture was transferred to a flask and heated to boiling reflux for 6 hours. After that, the solvent was evaporated and the obtained power samples were dried in an air-circulating oven at $140^{\circ} \mathrm{C}$ for 12 hours.

\section{Synthesis of $\mathrm{NaGdF}_{4}: \mathrm{Yb}, \mathrm{Tm} @ \mathrm{NaGdF}_{4}: \mathrm{Yb}$ (UCNPs)}

UCNPs were synthesized by a hot injection method. A total of $320 \mathrm{mg} \mathrm{Gd}\left(\mathrm{CF}_{3} \mathrm{COO}\right)_{3}, 50 \mathrm{mg} \mathrm{Yb}\left(\mathrm{CF}_{3} \mathrm{COO}\right)_{3}$, and $130 \mathrm{mg}$ $\mathrm{NaCF}_{3} \mathrm{COO}$ were dissolved in a mixture containing $3 \mathrm{~mL}$ $\mathrm{OA}$ and $3 \mathrm{~mL}$ ODE with stirring, and then the solution was heated to $130^{\circ} \mathrm{C}$ with magnetic stirring under vacuum for 30 minutes to remove dissolved oxygen and other impurities. After cooling to room temperature, the mixture was collected and marked as mixture A. Afterward, $400 \mathrm{mg} \mathrm{Gd}\left(\mathrm{CF}_{3} \mathrm{COO}\right)_{3}$, $50 \mathrm{mg} \mathrm{Yb}\left(\mathrm{CF}_{3} \mathrm{COO}\right)_{3}, 10 \mathrm{mg} \mathrm{Tm}\left(\mathrm{CF}_{3} \mathrm{COO}\right)_{3}$, and $130 \mathrm{mg}$ $\mathrm{NaCF}_{3} \mathrm{COO}$ were added into a flask and dissolved in $4 \mathrm{~mL}$ $\mathrm{OA}$ and $4 \mathrm{~mL} \mathrm{ODE}$, and heated to $130^{\circ} \mathrm{C}$ under vacuum for 30 minutes. Subsequently, the temperature was set to $320^{\circ} \mathrm{C}$ at $5^{\circ} \mathrm{C} / \mathrm{min}$ under the protection of Ar. Then mixture A was injected into the above system slowly and kept at $320^{\circ} \mathrm{C}$ for
1 hour. Finally, the product was collected with centrifugation at 16,000 rpm for 20 minutes and washed with absolute ethanol for several times.

\section{Synthesis of (UCNPs, C3)@PCL-PEG (PL-UC-C3)}

First, PEG-PCL (50 mg) was synthesized as per our previous report and dispersed in deionized water $(50 \mathrm{~mL}) .{ }^{18}$ Five milligrams of $\mathrm{C} 3$ and $5 \mathrm{mg}$ of UCNPs were dispersed in $10 \mathrm{~mL}$ dichloromethane and added into the above mentioned $50 \mathrm{~mL}$ suspension under ultrasonic stirring for 30 minutes. Then, the reaction system was heated to $70^{\circ} \mathrm{C}$ for 20 minutes to evaporate additional dichloromethane. Finally, the sample was separated by centrifugation at 6,000 rpm for 10 minutes, and washed with deionized water and absolute ethanol for several times.

\section{Characterization}

The morphological characteristics of the samples were observed by transmission electron microscopy (TEM, Model Tecnai 12; Philips Co., Ltd., Amsterdam, Netherlands). The size and size distribution of the samples were measured with dynamic light scattering (DLS, BI-90Plus; Brookhaven Instruments). The crystalline phases were determined by powder X-ray diffraction (XRD, Karlsruhe, Germany) using $\mathrm{Cu} \mathrm{K} \alpha$ radiation $(\lambda=1.5418 \AA$ ). The $U V$-vis absorbance spectra of the samples were recorded by UV-vis spectrophotometry (UV3100; Shimadzu, Tokyo, Japan). The up-conversion fluorescence of the sample was recorded by fluorescence instrument (FLS 980; Edinburgh, England).

\section{In vitro cytotoxicity assay (MTT assay)}

A MTT test was adopted to verify the cytotoxicity of PL-UC-C3. Briefly, 4T1 cells and HUVECs were incubated in a 96 -well plate $\left(5 \times 10^{3}\right.$ cells/well $)$ overnight, and then various concentrations of PL-UC-C3 mixed with $200 \mu \mathrm{L}$ of fresh culture medium were added into each well for 24 hours. After that, the medium was replaced with $200 \mu \mathrm{L}$ of fresh culture medium containing $20 \mu \mathrm{L}$ of MTT reagent $(5 \mathrm{mg} / \mathrm{mL})$ and incubated at $37^{\circ} \mathrm{C}$ for another 2 hours. Finally, the cell viability of each well was analyzed with an iMark Enzyme mark instrument (Bio-Rad Laboratories Inc., Hercules, CA, USA) by detecting the absorbance at $490 \mathrm{~nm}$. The data were treated from the average of three tests.

\section{Intracellular internalization analysis}

$4 \mathrm{~T} 1$ cells were seeded in a glass bottom dish and incubated at $37^{\circ} \mathrm{C}$ for overnight. After that, $50 \mu \mathrm{L}$ of PL-UC-C3 $(1 \mathrm{mg} / \mathrm{mL})$ was added, and the dish was placed in an incubator at $37^{\circ} \mathrm{C}$ for 
another 2 hours. Then the 4T1 cells were washed with PBS and stained with LysoTracker Red DND-99 (2 $\mu \mathrm{L}$ in $1 \mathrm{~mL}$ PBS). The treated samples were observed under a confocal laser scanning microscope (CLSM 700; Zeiss, Jena, Germany) with an excitation wavelength of $980 \mathrm{~nm}$ and an emission wavelength of $440 \mathrm{~nm}$ for signing PL-UC-C3, and LysoTracker Red DND-99 was treated with an excitation wavelength of $488 \mathrm{~nm}$ and an emission wavelength of $625 \mathrm{~nm}$ to mark lysosome.

\section{PTT study}

The mice bearing 4T1 tumor were injected with $200 \mu \mathrm{L}$ of products $(5 \mathrm{mg} / \mathrm{mL})$ via vena caudalis and anesthetized with $4 \%$ chloral hydrate at 8 hours post-injection. Then the mice were placed on an operating table and irradiated with a 980 $\mathrm{nm}$ laser $\left(500 \mathrm{~mW} / \mathrm{cm}^{2}\right)$, and the thermal images of the mice were recorded by a thermal imager (IRS 600; Fotric, Dalas, TX, USA). In addition, the thermal circulation ability of PLUC-C3 was detected in a small beaker $(5 \mathrm{~mL})$. Briefly, $1 \mathrm{mg}$ of PL-UC-C3 was dispersed in $1 \mathrm{~mL}$ of deionized water and transferred to the beaker. A $980 \mathrm{~nm}$ laser $\left(500 \mathrm{~mW} / \mathrm{cm}^{2}\right)$ was used to activate the samples until the temperature reached $45^{\circ} \mathrm{C}$, and after cooling to initial temperature, the operation was repeated for several times.

\section{Fluorescence imaging in vivo}

The nanocomposite was applied to diagnose tumor and exhibit the distribution in vivo using an imager (IVIS Spectrum; Perkin Elmer, Waltham, MA, USA). The 4T1 tumor-bearing mice were injected intravenously with $200 \mu \mathrm{L}$ of saline containing PL-UC-C3 (5 mg/mL), and the luminescence imaging was noted down by testing the sign at $650 \mathrm{~nm}$.

\section{Computed tomography imaging}

For CT imaging in vitro, different concentrations (1.25, $2.5,5,10$, and $20 \mathrm{mg} / \mathrm{mL}$, respectively) of PL-UC-C3 were stored in a 24-well plate and detected by a 64-Detector CT (Discovery CT 750HD; GE Amersham Healthcare System, Milwaukee, WI, USA). For CT imaging in vivo, the 4T1 tumor-bearing mice were intravenously injected with $200 \mu \mathrm{L}$ of PL-UC-C3 (20 mg/mL) and recorded by the 64-Detector CT 8 hours post-injection.

\section{Biodistribution of PL-UC-C3 NPs in vivo}

In order to investigate the degree of nanocomposite enrichment in tumor and primary organs, the mice bearing 4T1 tumor were intravenously injected with $200 \mu \mathrm{L}$ of PL-UC-C3 $(5 \mathrm{mg} / \mathrm{mL})$ and sacrificed at 1, 2, 4, 8, and 24 hours after the injection. Then the tumor and primary organs (heart, liver, spleen, lung, and kidney) were harvested and dissolved in the fresh aqua regia. After that, the biodistribution of PL-UC-C3 was quantified by an inductively coupled plasma mass spectrometry (Agilent 7,500 ce).

\section{In vivo antitumor effect}

$\mathrm{Balb} / \mathrm{c}$ mice were inoculated in subcutaneous right forelimb armpit with $4 \mathrm{~T} 1$ cells $\left(1 \times 10^{7}\right.$ per mouse $)$. While the tumor volume reached $100 \mathrm{~mm}^{3}$, 4T1 tumor-bearing mice were randomly divided into six groups with eight mice per group: saline group, $450 \mathrm{~nm}$ plus a $980 \mathrm{~nm}$ laser group, PL-C3 plus a $450 \mathrm{~nm}$ laser group, PL-C3 plus a 980 nm laser group, PL-UC-C3 plus a $450 \mathrm{~nm}$ laser group, and PL-UC-C3 plus a $980 \mathrm{~nm}$ laser group. The mice in saline group, PL-C3 plus a $450 \mathrm{~nm}$ laser group, PL-C3 plus a $980 \mathrm{~nm}$ laser group, PL-UC-C3 plus a $450 \mathrm{~nm}$ laser group, and PL-UC-C3 plus a $980 \mathrm{~nm}$ laser group were intravenously injected at a dose of $200 \mu \mathrm{L}(5 \mathrm{mg} / \mathrm{mL})$, and according to grouping, the mice were exposed to a $450 \mathrm{~nm}$ or $980 \mathrm{~nm}$ laser $\left(500 \mathrm{~mW} / \mathrm{cm}^{2}\right) 8$ hours after injection. Then, the tumor volume and body weight were recorded every day and the mice were sacrificed on 14th day; then the tumors were collected and fixed in 4\% paraformaldehyde. H\&E staining and ki67 staining of the tumors were implemented by Nanjing KeyGEN BioTECH Company and observed by a fluorescence microscope (XD-202; Jiang Nan, Nanjing, China). In addition, to study the histological changes in mice, the primary organs (heart, liver, spleen, lung, and kidney) were treated with PLUC-C3 plus a $980 \mathrm{~nm}$ laser, and were later made into sections and stained with $H \& E$ at different periods of time $(1,7,15$, and 30 days). Besides, the remaining mice were prepared to study the survival situation.

\section{Hematology and biochemical assay}

In order to measure the long-term cytotoxicity in mice treated with PL-UC-C3 plus a $980 \mathrm{~nm}$ laser, the blood was collected in a sodium EDTA anticoagulant tube for hematology assay. The total bilirubin, total protein, and the alanine aminotransferase levels were determined to assess the liver function. Creatinine level and blood urea nitrogen were used to evaluate the kidney function. The spleen function was evaluated by the production of platelets. The immune response was evaluated by testing lymphocytes, neutrophils, and monocytes, while behavioral approach system, eosinophils, and red blood cell levels were leveraged to assess the cytotoxicity of treatment process.

\section{Statistical analysis}

All the data in this work were obtained from the average of experiments conducted in triplicate and analyzed via Student's $t$-test by leveraging the GraphPad Prism (version 7.5) 
software at a significance level of $P<0.05$. The significant differences are marked by asterisks.

\section{Results and discussion Characterization of PL-UC-C3}

The morphology and size of the products were characterized by TEM. As shown in Figure 2A and B, both UCNPs and
PL-C3 NPs presented a homogeneously dispersed morphology with a size of about $16 \mathrm{~nm}$ and $60 \mathrm{~nm}$, respectively. After the encapsulation of UCNPs into PL-C3 NPs to acquire PL-UC-C3 NPs, uniformly spherical and mono-dispersed NPs could be noticed in the TEM micrographs (Figure 2C) with a mean diameter of $80 \mathrm{~nm}$, which is larger than the size of PL-C3 NPs, thereby robustly indicating the effective
A

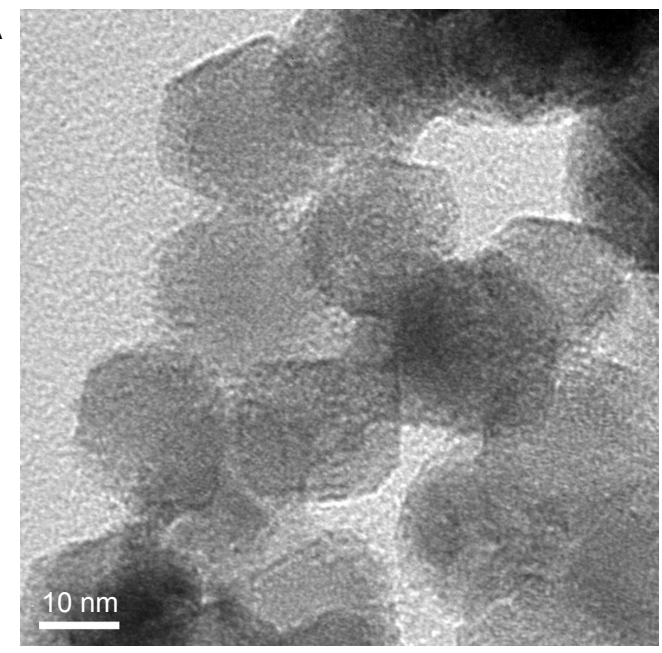

C
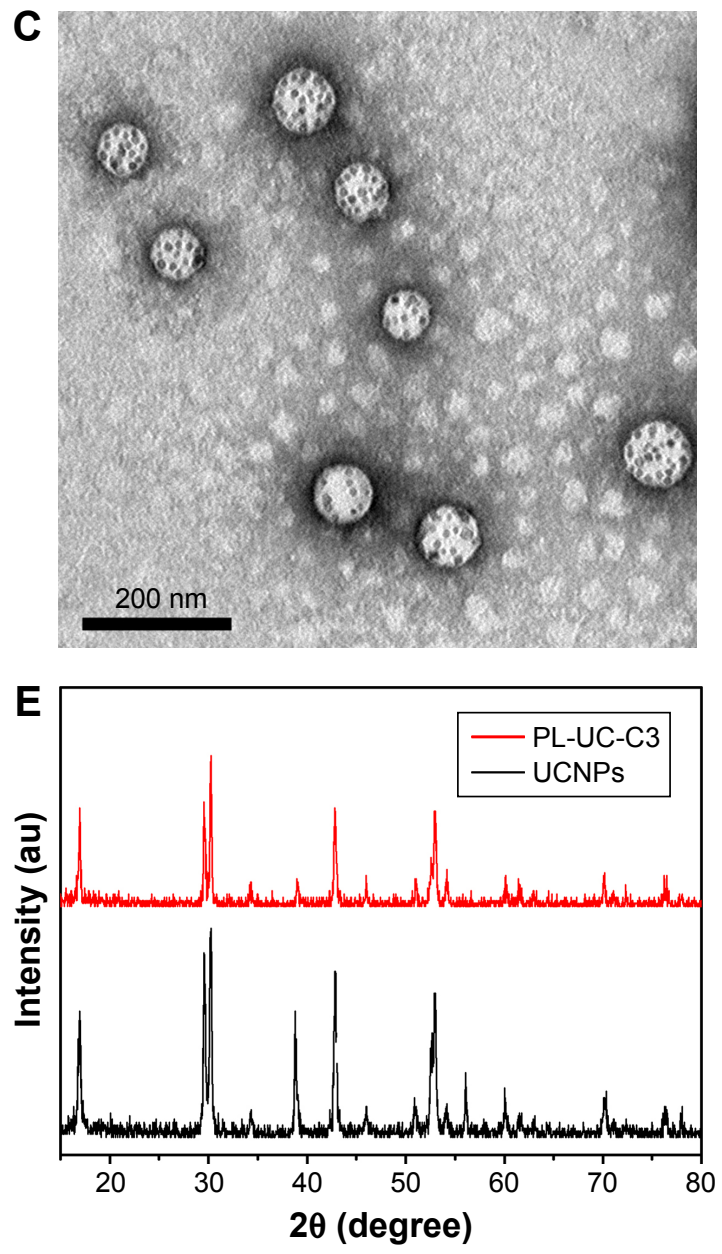

B

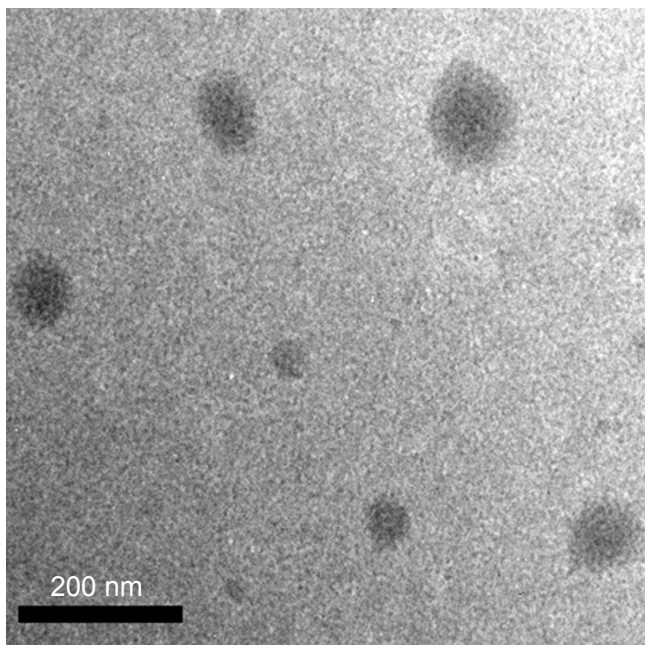

D

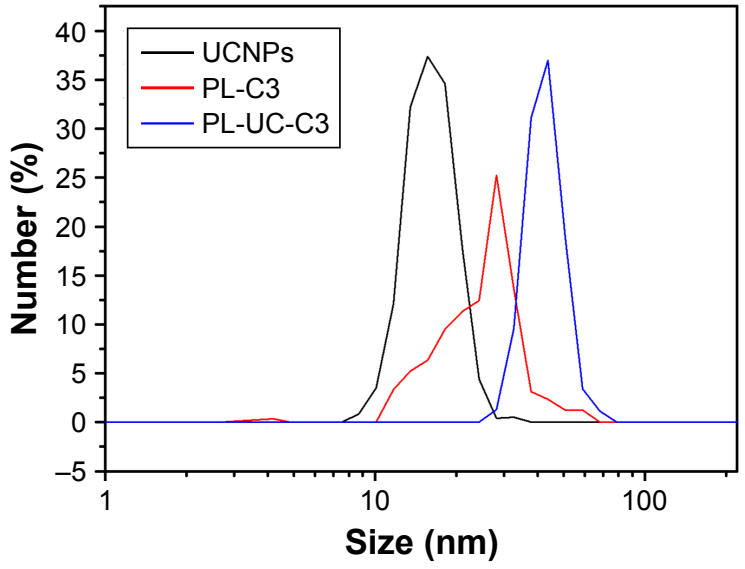

F



Figure 2 (Continued) 

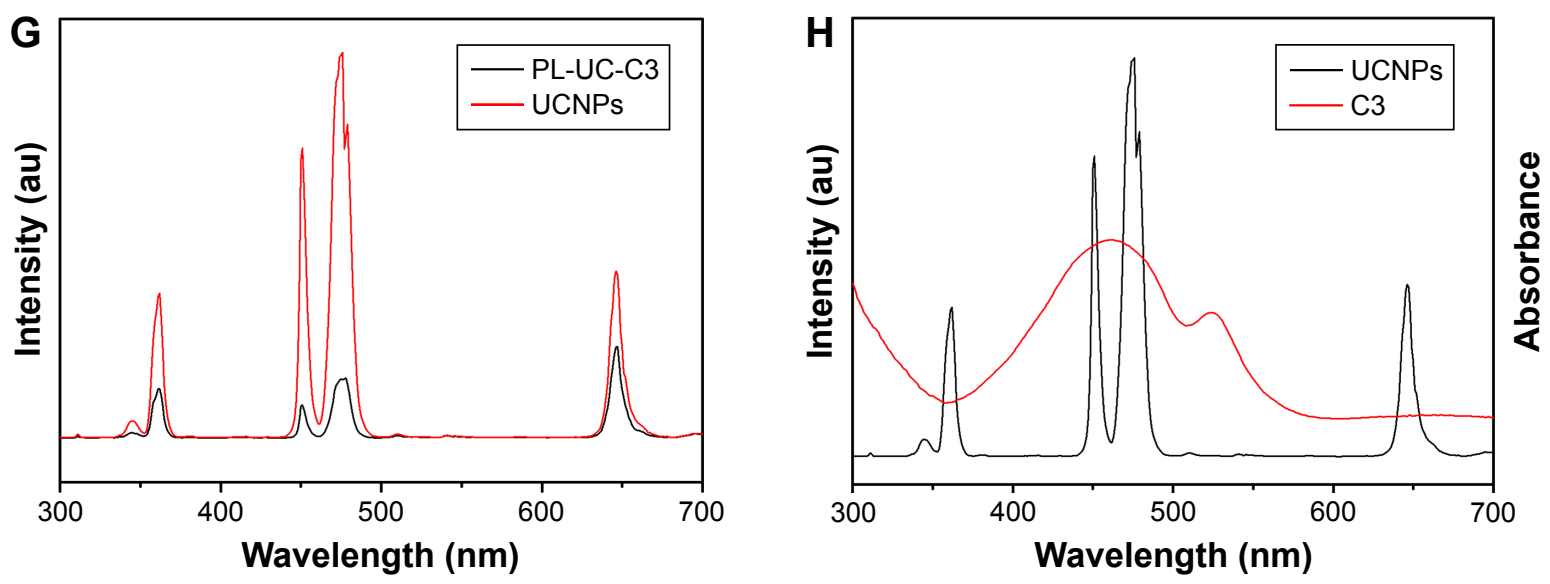

Figure 2 (A) TEM image of UCNPs in ethanol. (B) TEM image of PL-C3 in deionized water. (C) TEM image of PL-UC-C3 in deionized water. (D) DLS analysis of UCNPs, PL-C3, and PL-UC-C3. (E) Powder XRD diffraction patterns of UCNPs and PL-UC-C3. (F) UV-vis spectra of UCNPs, C3, and PL-UC-C3 (I mg/mL all). (G) UP-conversion emission spectra of UCNPs and UV-vis spectrum of C3. (H) Up-conversion emission spectra of UCNPs and PL-UC-C3.

Note: C3, organic compound; PL-UC-C3, encapsulation of UCNPs and C3 into PEG-PCL; PL-C3, encapsulation of C3 into PEG-PCL.

Abbreviations: UCNPs, up-conversion nanoparticles; TEM, transmission electron microscopy; DLS, dynamic light scattering; XRD, X-ray diffraction; UV-vis, ultravioletvisible; PEG, polyethylene-polyglycol; PCL, poly-e-caprolactone.

encapsulation of UCNPs. The corresponding size distribution was measured through the investigation of hydrodynamic diameter using the DLS. From Figure 2D, a slight increase in hydrodynamic diameter compared to TEM data and a narrow size distribution are clearly depicted. The XRD spectra of UCNPs and PL-UC-C3 are presented in Figure 2E. Compared with the standard hexagonal- $\mathrm{NaGdF}_{4}$ (JCPDS 027-0699), the corresponding peaks were observed in UCNPs and PL-UC-C3, indicating the successful synthesis of UCNPs and the successful encapsulation of UCNPs in PC-UC-C3. Furthermore, to ensure the successful encapsulation of $\mathrm{C} 3$ in PC-UC-C3, UV-vis absorption spectra of relevant nanoparticles were tested (Figure 2F). Quite evidently, similar absorption peaks of $\mathrm{C} 3$ were detected in the curve of PL-UC-C3 NPs, illustrating that C3 was successfully integrated in PCLPEG. The up-conversion emissions of PL-UC-C3 and UCNPs were detected by exciting with a $980 \mathrm{~nm}$ laser. As illustrated in Figure 2G, the maximum intensity of UCNPs was found at 440 and $470 \mathrm{~nm}$, which exactly matched the absorption peaks of $\mathrm{C} 3$, suggesting that the stimulated luminescence could be absorbed by $\mathrm{C} 3$ for PTT. Furthermore, the vast majority of luminous energy (less than $600 \mathrm{~nm}$ ) emitted by UCNPs was absorbed by $\mathrm{C} 3$ while up-conversion emissions at $650 \mathrm{~nm}$ was negligibly affected, which confirmed that the tumor could be detected through fluorescence diagnosis at $650 \mathrm{~nm}$ (Figure 2H).

\section{In vitro biocompatibility and cytotoxicity profiles}

Intelligent and smart nanoparticles featuring low cytotoxicity and excellent biocompatibility were a critical and essential precondition for the in vivo application. First, an intracellular internalization experiment was performed. As observed in Figure 3A, red-marked (625 nm) lysosome with an excitation wavelength of $488 \mathrm{~nm}$ and blue-marked (440 nm) PL-UC-C3 with an excitation wavelength of $980 \mathrm{~nm}$ were perfectly merged, indicating that the nanocomposites were swallowed by cells via endocytosis. Next, the biocompatibility and cell toxicity of PL-UC-C3 were tested using cancer cell (4T1) and normal cell (HUVEC) lines, respectively; as reflected in Figure $3 \mathrm{~B}$ and $\mathrm{C}$, the cell viability was sustained at high levels when co-cultured with PL-UC-C3 at low concentrations. Furthermore, 80\% of the cells survived even when the concentrations reached $2,000 \mu \mathrm{g} / \mathrm{mL}$, which fully demonstrated the good biocompatibility of PL-UC-C3.

\section{PTT efficacy}

A crucial issue in PTT treatment is the light-thermal conversion efficiency. Hence, a rational design composed of maximum light absorption and deep tumor penetration should be judiciously considered and exploited. In this platform, a $980 \mathrm{~nm}$ laser $\left(500 \mathrm{~mW} / \mathrm{cm}^{2}\right)$ was adopted to irradiate PL-UC-C3 NPs for PTT treatment, and we hypothesized that the introduction of UCNPs would bring a phenomenal advancement in hyperthermia. From Figure 4A, we could find that compared with saline group or laser alone group, PL-C3 group only obtained a small increase in temperature, regardless of the irradiation by a $980 \mathrm{~nm}$ or $450 \mathrm{~nm}$ laser mainly because of the ignorable absorption of $980 \mathrm{~nm}$ laser by $\mathrm{C} 3$ and the low penetration of $450 \mathrm{~nm}$ laser. Notably, the additional engagement of UCNPs was game changing 
A

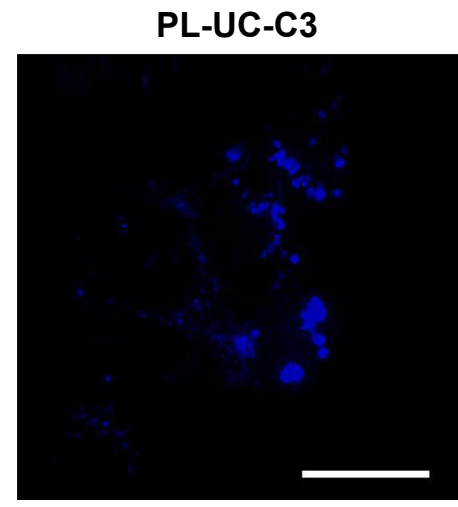

B

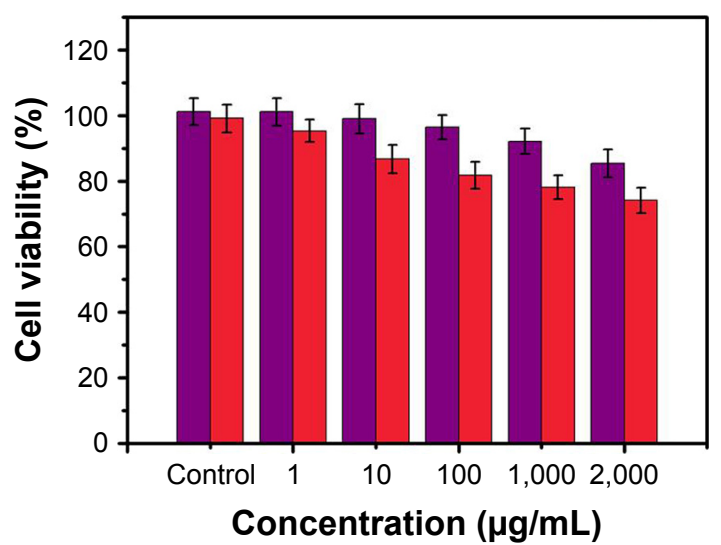

Lysosome

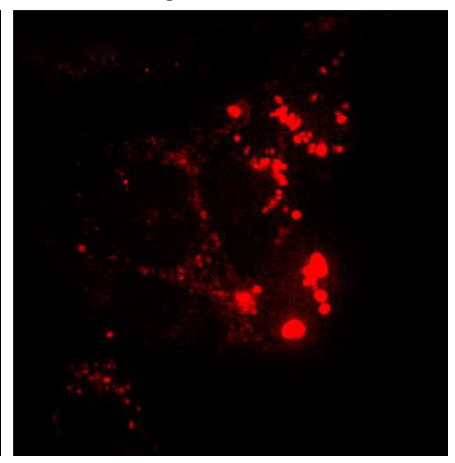

C

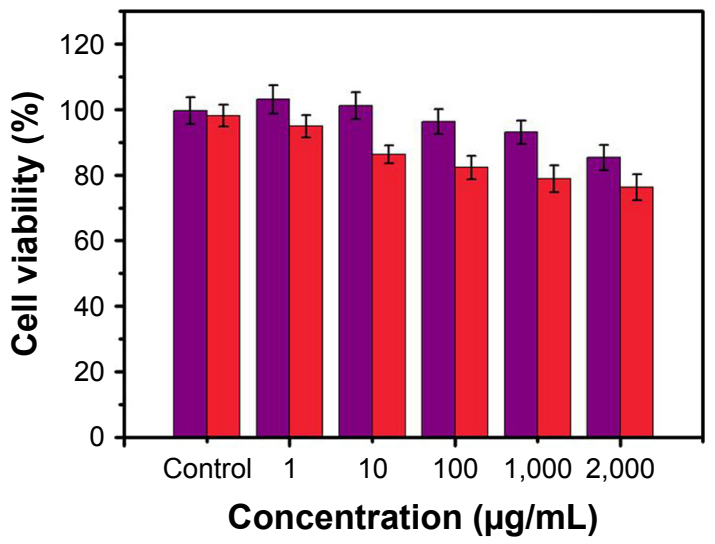

$24 \mathrm{~h} \square 48 \mathrm{~h}$

Figure 3 (A) Endocytosis of PL-UC-C3 in 4TI cells. LysoTracker Red DND-99 (red, $E_{x}=488 \mathrm{~nm}, \mathrm{E}_{\mathrm{m}}=625 \mathrm{~nm}$ ) was used to mark the lysosome, while blue was employed to indicate the trace of PL-UC-C3 $\left(E_{x}=980 \mathrm{~nm}, E_{m}=440 \mathrm{~nm}\right)$. The scale bar is $10 \mu \mathrm{m}$. In vitro cell viability of (B) 4TI cells and (C) HUVECs incubated with PL-UC-C3 without irradiation.

Note: C3, organic compound; PL-UC-C3, encapsulation of UCNPs and C3 into PEG-PCL; PL-C3, encapsulation of C3 into PEG-PCL.

Abbreviations: HUVECs, human umbilical vein endothelial cells; PEG, polyethylene-polyglycol; PCL, poly-e-caprolactone; UCNPs, up-conversion nanoparticle.

and PL-UC-C3 displayed a significant temperature change under the irradiation of a $980 \mathrm{~nm}$ laser owing to the highly efficient absorption and transformation ability of light. The corresponding time-temperature curves of above mentioned groups are recorded in Figure 4B, from which we could notice that the temperature in tumor site increased uninhibitedly and reached $46.6^{\circ} \mathrm{C}$ under the irradiation of a $980 \mathrm{~nm}$ laser for 10 minutes, supplying a hyperthermia beyond the tolerance of cancer cells. In contrast, in other group without the addition of UCNPs or without the help of a $980 \mathrm{~nm}$ laser, the temperature increased slowly and the tumor would eventually survive the sublethal hyperthermia. Besides, excellent thermal circulation ability was confirmed (Figure 4C). Taken together, PL-UC-C3 NPs have the promising potential for the cascade conversion PTT treatment.

\section{Fluorescence imaging analysis}

Fluorescence imaging was carried out to confirm the in vivo distribution of PL-UC-C3. The mice bearing 4T1 tumor were sacrificed 8 hours after the injection. As shown in Figure 5A, the fluorescence signal was detected in tumor site, proving the highly effective enrichment of nanoparticles in tumor tissues. Subsequently, the tumor and major organs were harvested and fluorescence imaging was conducted 8 hours after the injection (Figure 5B), and an extremely strong fluorescence signal was detected in tumor while relatively weak fluorescence signals were detected in liver and spleen, which was identical with the data of in vivo fluorescence imaging. All the above mentioned results indicated that PL-UC-C3 could be enriched in tumor site and play an indispensable role in guiding the tumor treatment.

\section{CT imaging detection}

$\mathrm{CT}$ as a very robust and smart imaging tool was employed to guild the PTT treatment rendered by PL-UC-C3 NPs. As observed in Figure 6A and B, a linear relationship was obtained between intensity and concentration, and with the increase of the concentration, the contrast ratio of material 

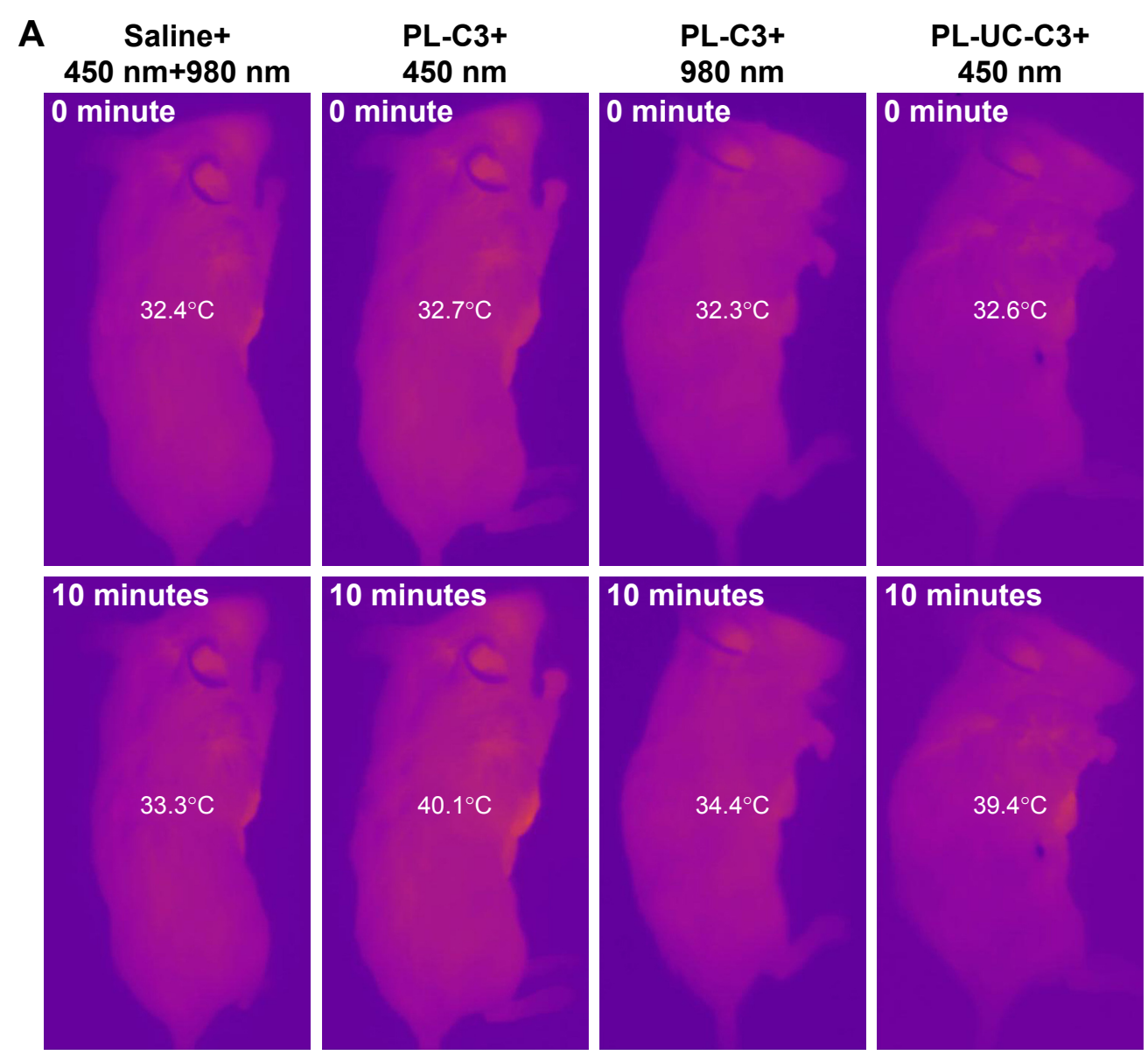

B

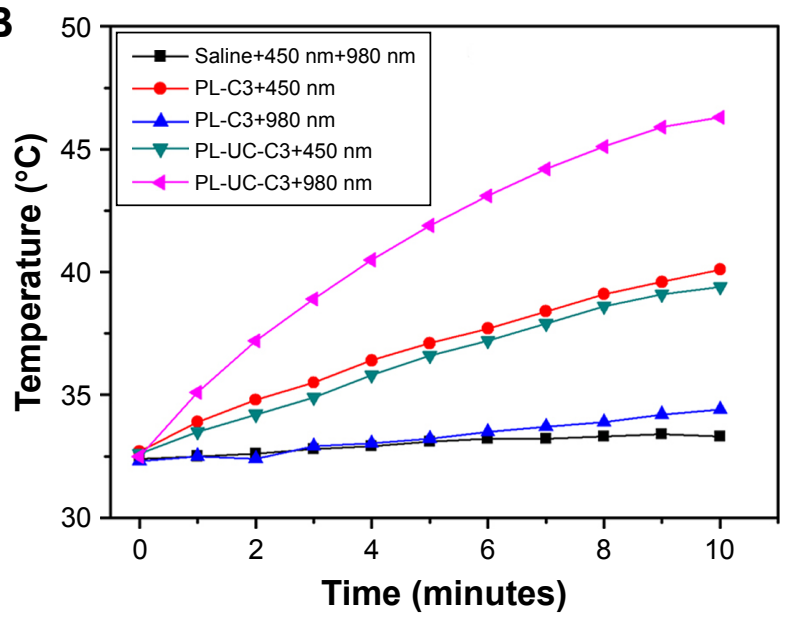

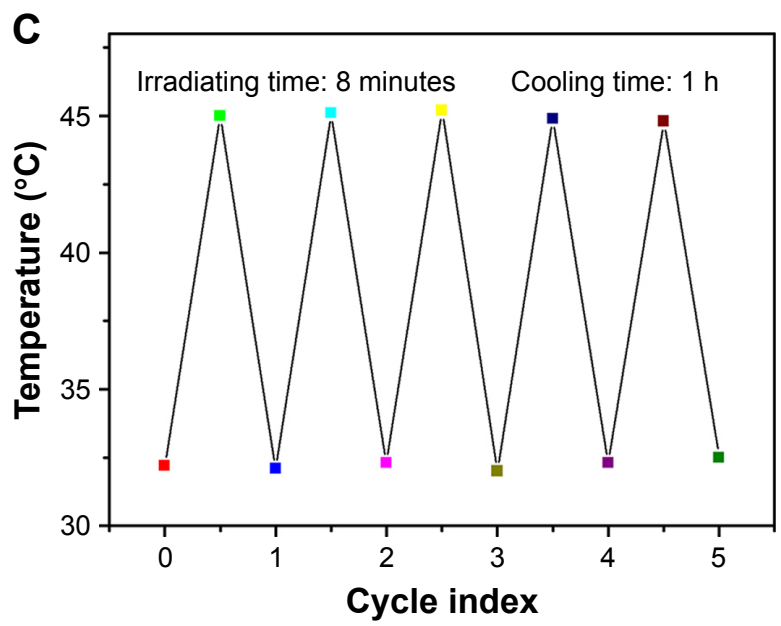

Figure 4 (A) Photothermal imaging of 4TI tumor-bearing mice intravenously injected with saline, PL-C3, or PL-UC-C3 after irradiation with a 450 nm or 980 nm $\left(500 \mathrm{~mW} / \mathrm{cm}^{2}\right)$ laser for 10 minutes. (B) The time-temperature curve of A. (C) Temperature of PL-UC-C3 NP solution (I mg dispersed in I mL of deionized water) after five cycles of NIR laser irradiation $\left(500 \mathrm{~mW} / \mathrm{cm}^{2}, 8\right.$ minutes each time)/cooling down (I h) process.

Note: C3, organic compound; PL-UC-C3, encapsulation of UCNPs and C3 into PEG-PCL; PL-C3, encapsulation of C3 into PEG-PCL.

Abbreviations: PEG, polyethylene-polyglycol; PCL, poly-e-caprolactone; UCNPs, up-conversion nanoparticles; NIR, near infrared.

and background was increased rapidly. As depicted in Figure 6C, a moderate $\mathrm{CT}$ signal was detected in tumor position 8 hours after the intravenous injection, while no detective CT signal was found in control group, clarifying the feasibility of PL-UC-C3 NPs as CT agents to mark the tumor and guide the PTT treatment.

\section{Biodistribution analysis}

After the injection of $200 \mu \mathrm{L}$ of PL-UC-C3 $(5 \mathrm{mg} / \mathrm{mL})$, tumor and primary organs were harvested to analyze the distribution condition of Gd. As seen in Figure 7, PL-UC-C3 was initially concentrated in liver, and as time went by, the nanocomposites were increasingly enriched in tumor with 

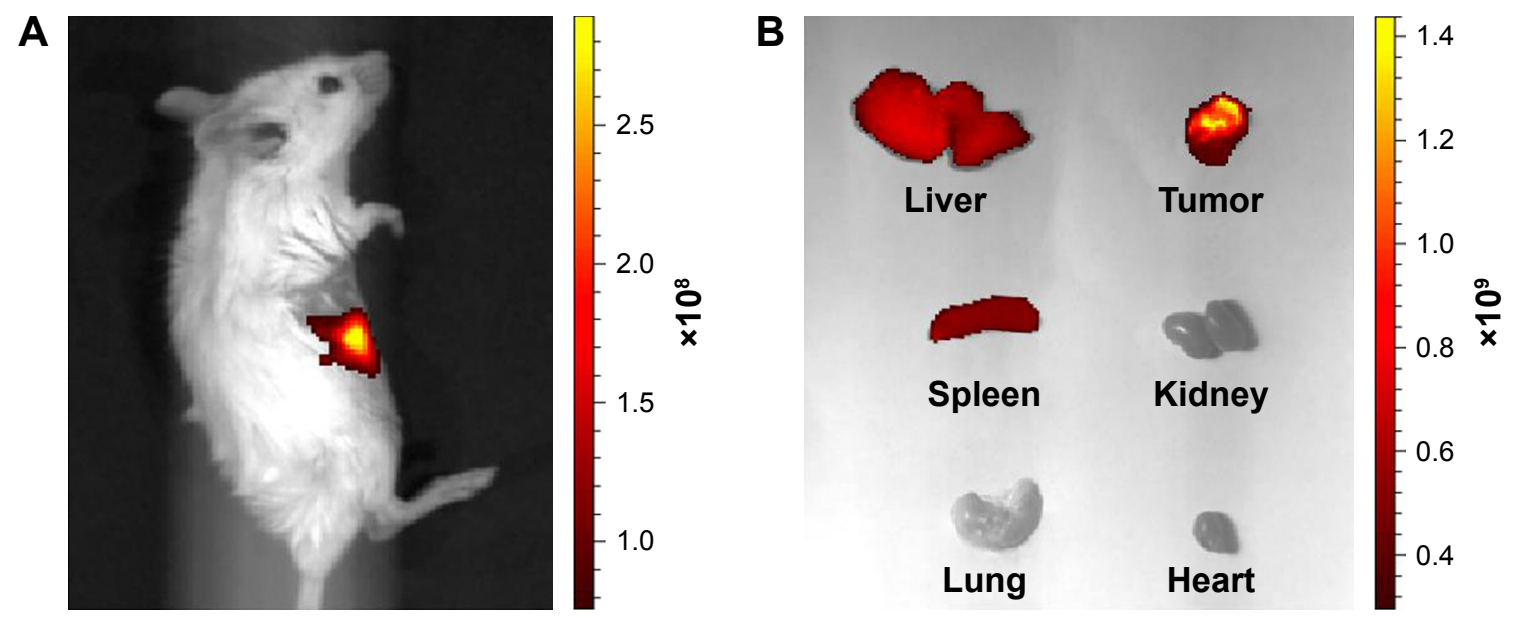

Figure 5 (A) In vivo fluorescence imaging of PL-UC-C3 after injection for 8 hours. (B) Fluorescence imaging of the tumor and primary organ (heart, liver, spleen, lung, and kidney) after injection for 8 hours.

Note: C3, organic compound; PL-UC-C3, encapsulation of UCNPs and C3 into PEG-PCL.

Abbreviations: PEG, polyethylene-polyglycol; PCL, poly-e-caprolactone; UCNPs, up-conversion nanoparticles.

the Gd content decreasing in liver. The result was coincident with that of fluorescence imaging and CT imaging, suggesting that PL-UC-C3 displayed a typical enhanced permeability and retention effect. ${ }^{29-31}$

\section{In vivo antitumor effect}

To verify the therapeutic effect of PL-UC-C3 on a 4T1 tumor-bearing mouse model, saline or nanocomposites were intravenously injected. The body weight and tumor volume were recorded on day 14 of the treatment period, and the survival cycle of mice was also studied for a longer period of time. Obviously, the group treated with PL-UC-C3 under a $980 \mathrm{~nm}$ laser irradiation exhibited a great inhibition function in tumor size (Figure 8A), while the tumors rapidly increased in the saline and NIR laser groups, and for PL-C3 plus a $450 \mathrm{~nm}$ laser group, PL-C3 plus a $980 \mathrm{~nm}$ laser group, and PL-UC-C3 plus a $450 \mathrm{~nm}$ group, the tumor volumes grew slowly at the beginning of treatment, but the increase rate of tumor volume gradually recovered on day 10. Moreover, the subacute toxicity of material was analyzed through the body weight detection. As shown in Figure 8B, there was no obvious change in body weight of mice, indicating innocuity of the nanocomposites. Furthermore, the survival cycle of 4T1 tumor-bearing mice is
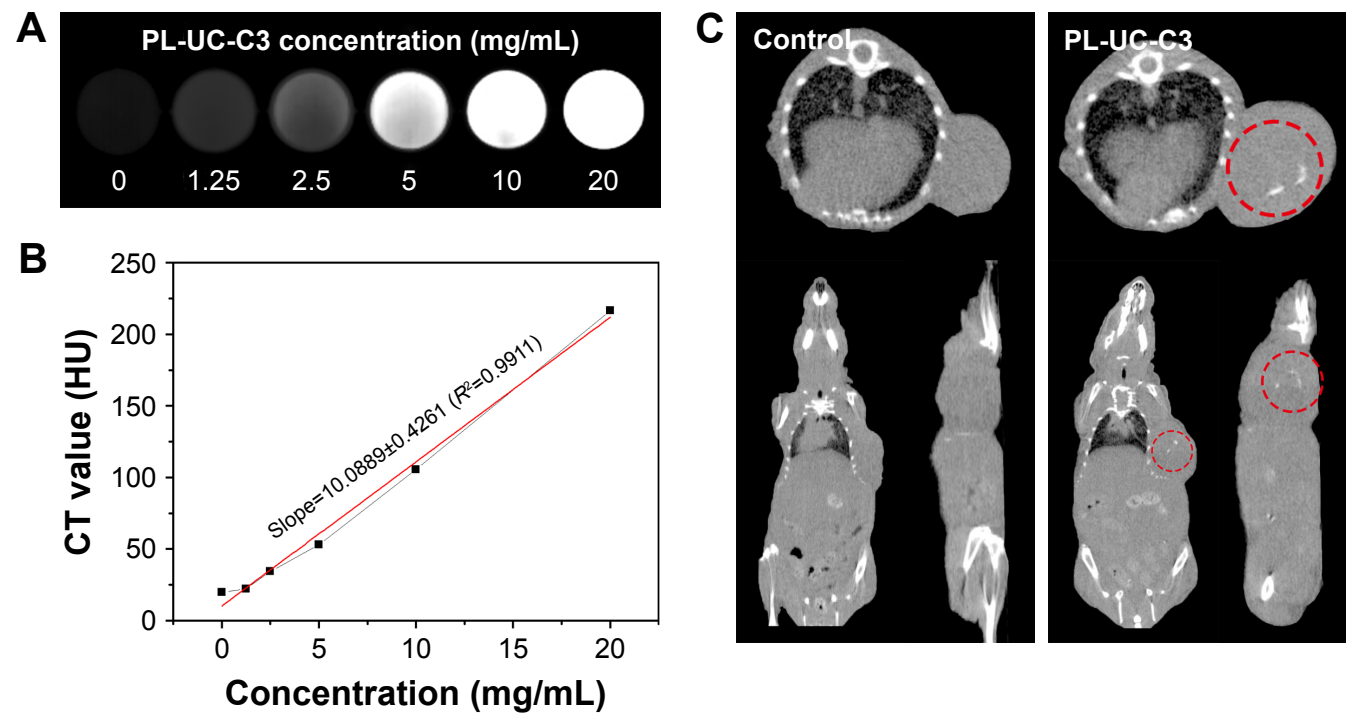

Figure 6 (A) In vitro CT imaging of PL-UC-C3 with different concentrations (0, I.25, 2.5, 5, I0, and $20 \mathrm{mg} / \mathrm{mL})$ in 24-well plates. (B) The corresponding linear relation curve of PL-UC-C3 between concentration and intensity. (C) In vivo CT imaging of PL-UC-C3 8 hours after the injection. The red circles depict the tumor positions. Note: C3, organic compound; PL-UC-C3, encapsulation of UCNPs and C3 into PEG-PCL.

Abbreviations: CT, computed tomography; PEG, polyethylene-polyglycol; PCL, poly-e-caprolactone; UCNPs, up-conversion nanoparticles. 


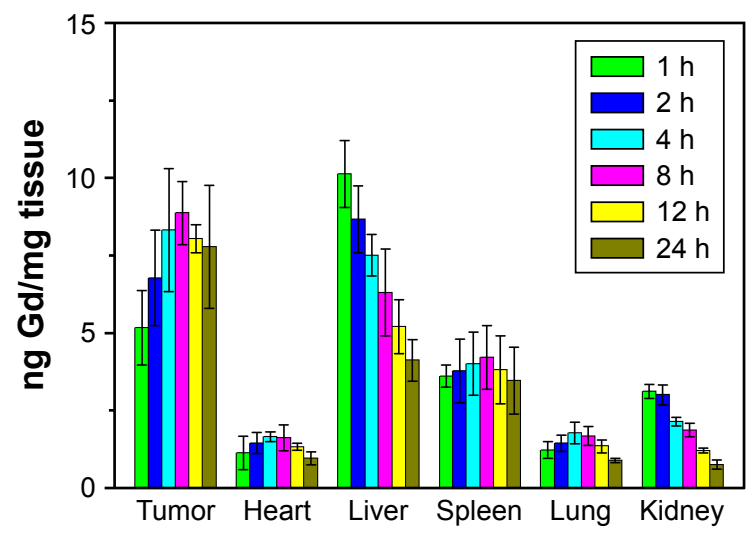

Figure $\mathbf{7}$ In vivo distribution profiles of PL-UC-C3 in tissues of 4TI tumor-bearing mice at different time points post-intravenous injection.

Note: C3, organic compound; PL-UC-C3, encapsulation of UCNPs and C3 into PEG-PCL.

Abbreviations: PEG, polyethylene-polyglycol; PCL, poly-e-caprolactone; UCNPs, up-conversion nanoparticles.

presented in Figure 8C, which shows that all the mice that did not undergo treatment died at different time points, while there was no case of death in PL-UC-C3 plus a $980 \mathrm{~nm}$ laser group. Besides, the representative tumor pictures are

A

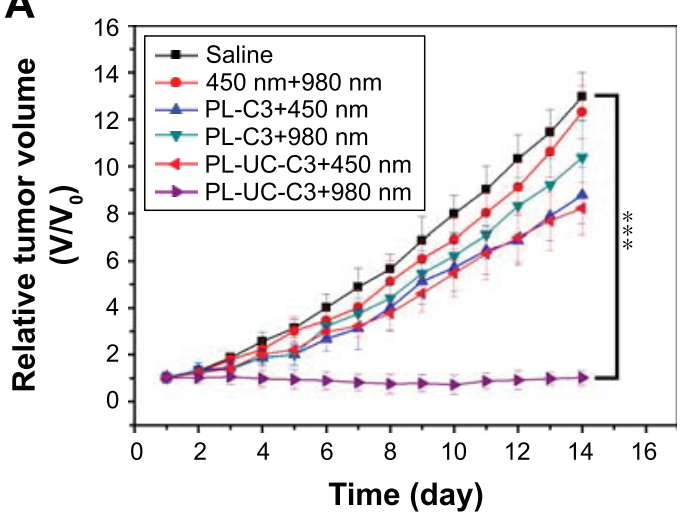

C

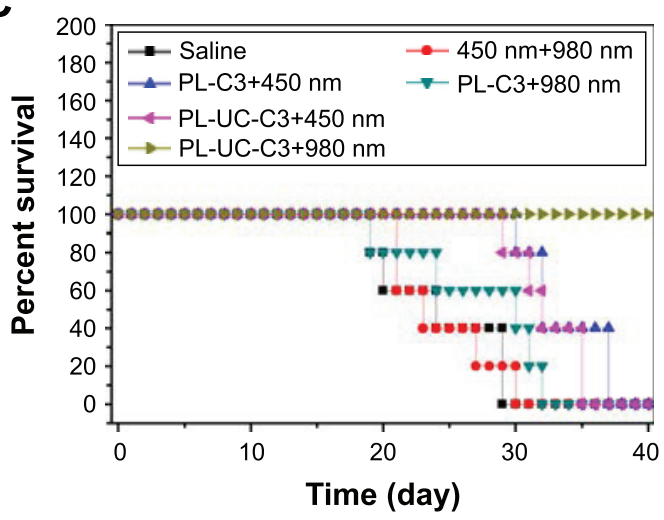

presented in Figure 8D, from which we could conclude that PL-UC-C3 exhibited the most superior PTT treatment effect against 4T1 tumor.

After receiving different treatments, the mice were sacrificed and the tumors were collected. The H\&E and ki67 staining assays were conducted to analyze the treatment effect of PTT. As analyzed by H\&E staining in Figure 9A, the tissue morphology of tumor was in good condition and no obvious necrotic and apoptotic areas were observed in saline and laser irradiation group. Contrarily, a spot of necrotic and apoptotic area was observed in PL-C3 plus a $450 \mathrm{~nm}$ laser group, PL-C3 plus a $980 \mathrm{~nm}$ laser group, and PL-UC-C3 plus a $450 \mathrm{~nm}$ laser group, while a large amount of necrosis (red arrows) and apoptosis (green arrows) were detected in PL-UC-C3 plus a $980 \mathrm{~nm}$ laser group. Ki67 staining analysis and the quantification for Ki67 staining were further used to study the cell's proliferation and differentiation (Figure 9B and C). A large amount of tumor proliferation was observed in the control group, while the game was changing in PL-UC-C3 plus a $980 \mathrm{~nm}$ laser group and only a slight tumor proliferation

B

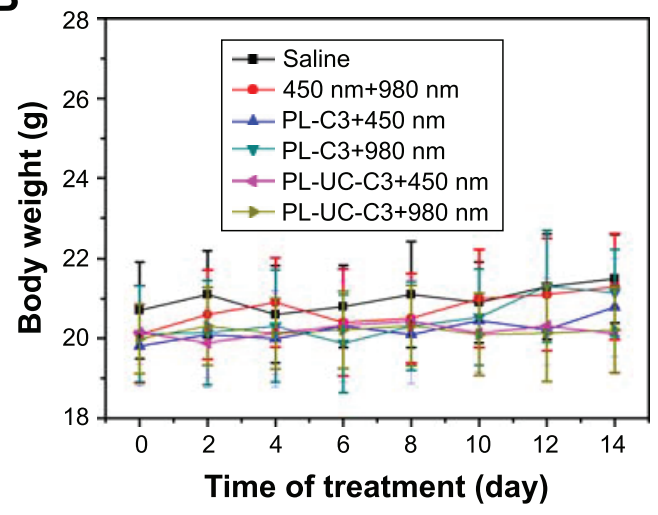

D

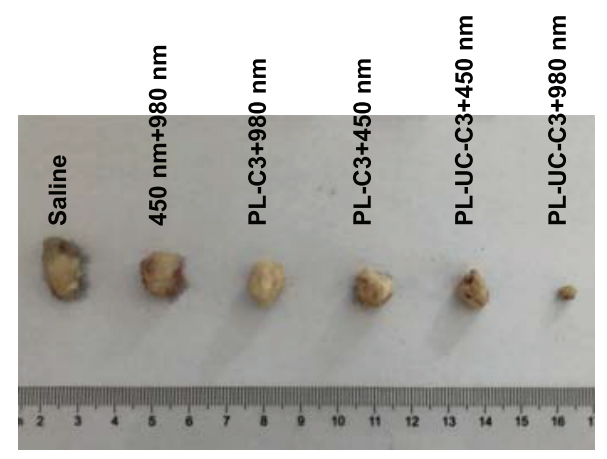

Figure 8 (A) Tumor volumes of 4TI tumor-bearing mice that received different treatments as indicated. The data are presented as mean \pm SD ( $n=8$ ). $* * * P<0.00 I$. (B) Body weight of $4 \mathrm{TI}$ tumor-bearing mice that received different treatments as displayed. (C) Survival curves of $4 \mathrm{TI}$ tumor-bearing mice that received different treatments as indicated. (D) Photographs of tumors dissected from mice that received different treatments as displayed.

Note: C3, organic compound; PL-UC-C3, encapsulation of UCNPs and C3 into PEG-PCL; PL-C3, encapsulation of C3 into PEG-PCL.

Abbreviations: PEG, polyethylene-polyglycol; PCL, poly-e-caprolactone; UCNPs, up-conversion nanoparticles. 


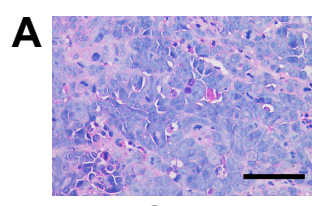

Saline

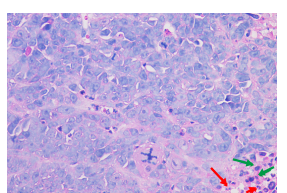

PL-C3+450 nm

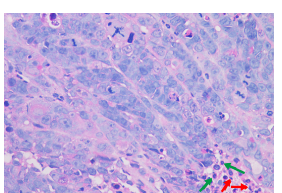

PL-UC-C3+450 nm

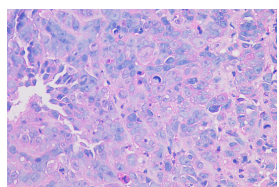

$450 \mathrm{~nm}+980 \mathrm{~nm}$

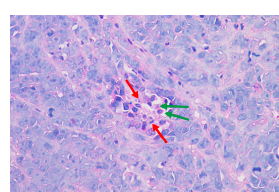

$\mathrm{PL}-\mathrm{C} 3+980 \mathrm{~nm}$



PL-UC-C3+980 nm

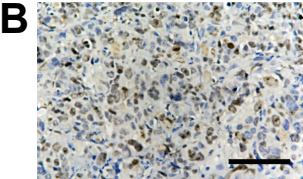

Saline

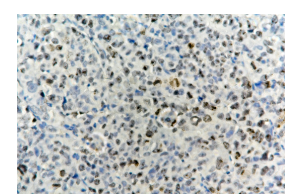

$\mathrm{PL}-\mathrm{C} 3+450 \mathrm{~nm}$

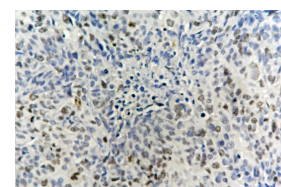

PL-UC-C3+450 nm

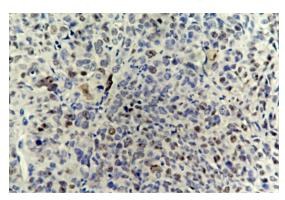

$450 \mathrm{~nm}+980 \mathrm{~nm}$

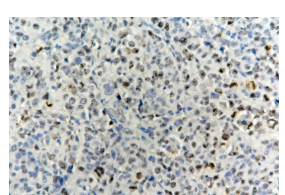

$\mathrm{PL}-\mathrm{C} 3+980 \mathrm{~nm}$

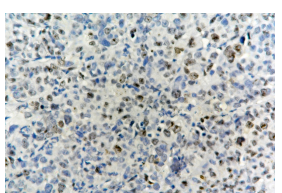

PL-UC-C3+980 nm

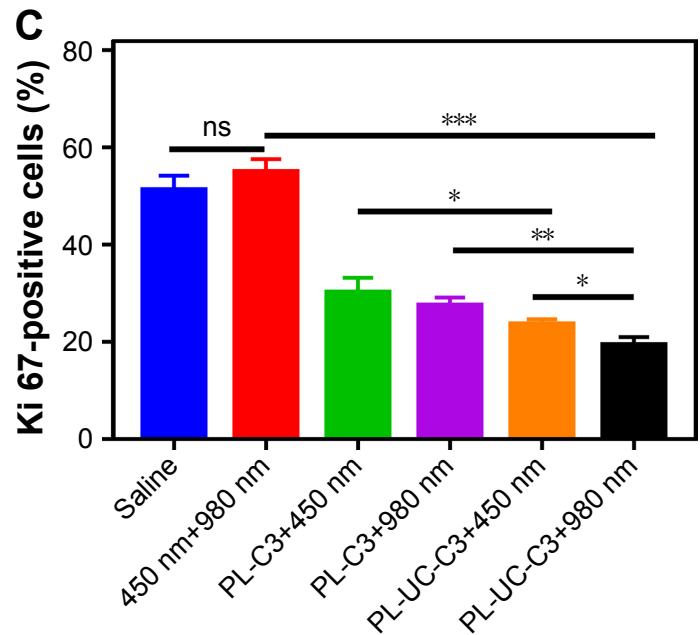

Figure 9 (A) H\&E staining of 4TI tumors after different treatments (red arrow: necrosis; green arrow: apoptosis). The scale bar is $50 \mu \mathrm{m}$. (B) Ki67 staining of 4TI tumors after different treatments. The scale bar is $50 \mu \mathrm{m}$. (C) The quantification study for Ki67 staining of $4 \mathrm{TI}$ tumors after different treatments. $* P<0.05$, $* * P<0.0 \mathrm{I}$, $* * * P<0.00 \mathrm{I}$. Note: C3, organic compound; PL-UC-C3, encapsulation of UCNPs and C3 into PEG-PCL; PL-C3, encapsulation of C3 into PEG-PCL.

Abbreviations: PEG, polyethylene-polyglycol; PCL, poly-e-caprolactone; UCNPs, up-conversion nanoparticles; ns, not significant.

was presented, again implying that cascade amplified hyperthermia rendered by PL-UC-C3 plus a $980 \mathrm{~nm}$ laser for tumor treatment was superior to other groups.

\section{Long-term cytotoxicity}

In order to confirm the safety of cascade amplified PTT rendered by PL-UC-C3 plus a $980 \mathrm{~nm}$ laser to the body, a histological and hematology analysis was conducted. For histological analysis, the organs were treated with H\&E staining. As shown in Figure 10, no obvious harm was found after treating with hyperthermia rendered by PL-UC-C3 plus a $980 \mathrm{~nm}$ laser in different time periods by contrast with untreated group. For the hematology analysis (Figure 11), we could find that despite indexes being unstable in the initial stage, they returned to normal level after a week. All the results of this study illustrated that the harmless PL-UC-C3 NPs could have greater prospects for application in tumor treatment and diagnosis.

\section{Conclusion}

We tailor-made PL-UC-C3 NPs which could efficiently absorb the energy of a $980 \mathrm{~nm}$ laser, transform them into a visible light, and deliver the light to $\mathrm{C} 3$, thus accomplishing a cascade amplified hyperthermia. The usage of a $980 \mathrm{~nm}$ laser confirms a deeper penetration and lower energy, while the highly efficient absorption and transformation process offers a phenomenal hyperthermia for tumor treatment. Finally, florescence/CT-guided PTT treatment successfully eliminated 4T1 tumor, throwing a light on its clinical application in cancer treatment. 

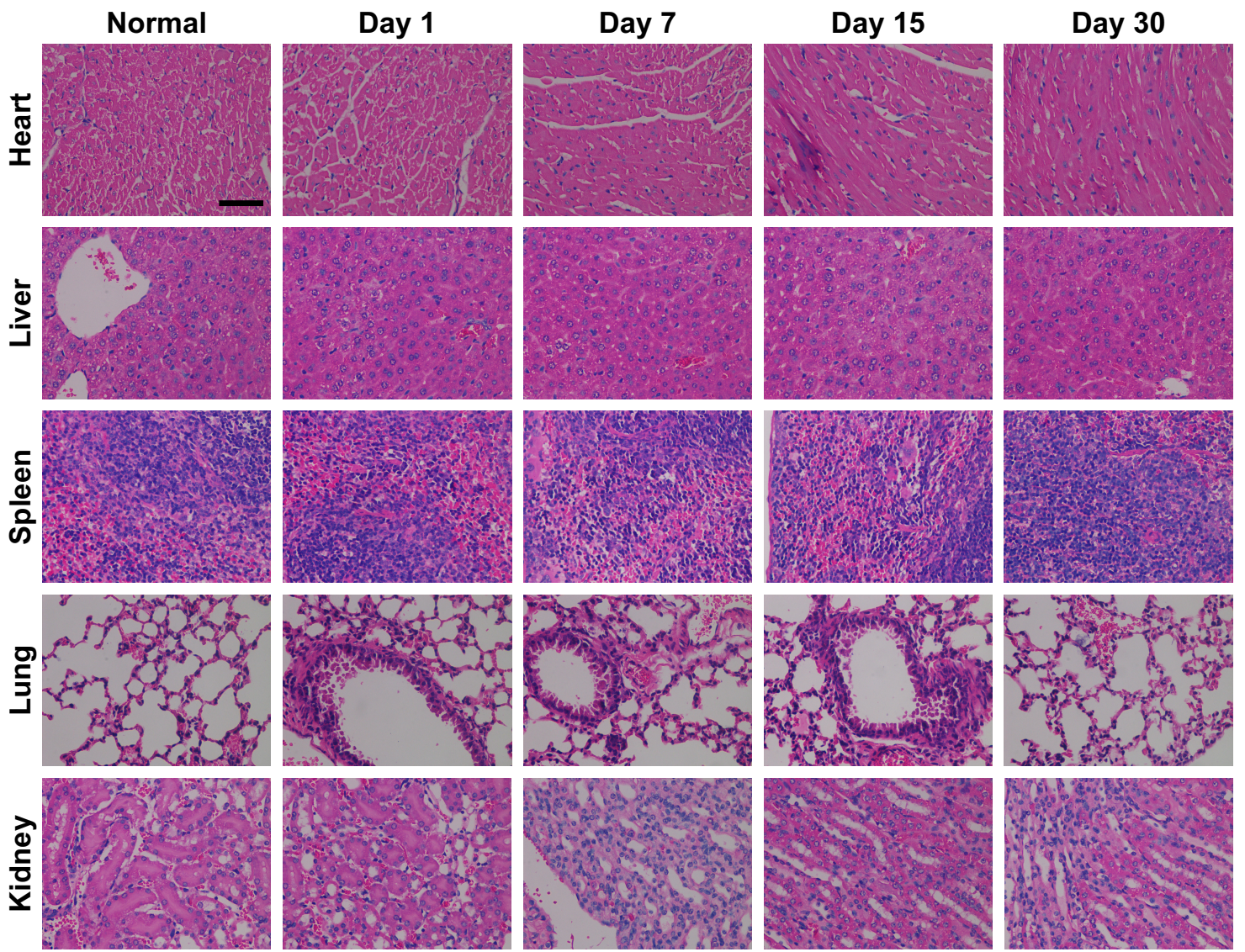

Figure 10 Studies of the long-term toxicity of the cascade amplified PTT treatment rendered by PL-UC-C3 plus a $980 \mathrm{~nm}$ laser through the H\&E staining of heart, liver, spleen, lung, and kidney for I, 7, I5, and 30 days after the treatment.

Note: C3, organic compound; PL-UC-C3, encapsulation of UCNPs and C3 into PEG-PCL; PTT, photothermal therapy.

Abbreviations: PEG, polyethylene-polyglycol; PCL, poly-e-caprolactone; UCNPs, up-conversion nanoparticles.

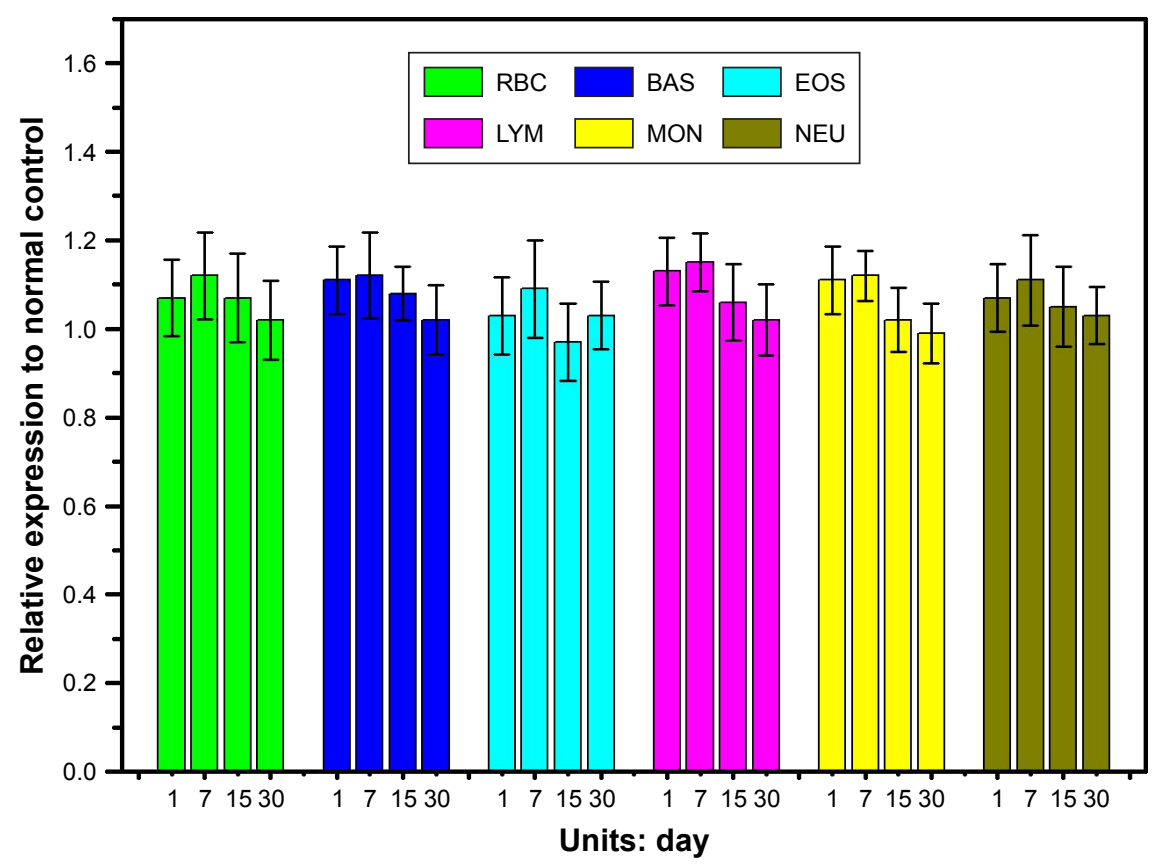

Figure I I (Continued) 


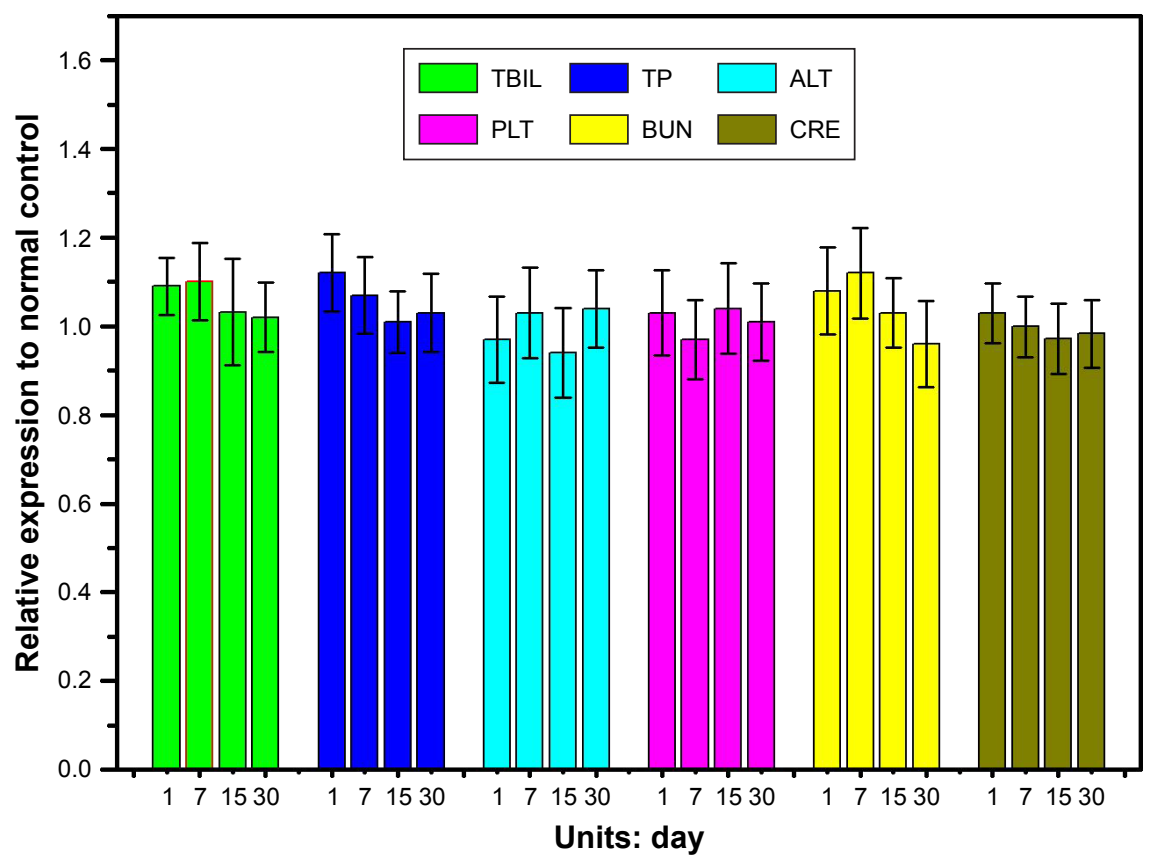

Figure I I Serum biochemical study I, 7, I5, and 30 days after the cascade amplified PTT treatment rendered by PL-UC-C3 plus a $980 \mathrm{~nm}$ laser ( $\mathrm{n}=3$ at each time point). Note: C3, organic compound; PL-UC-C3, encapsulation of UCNPs and C3 into PEG-PCL.

Abbreviations: PEG, polyethylene-polyglycol; PCL, poly-e-caprolactone; UCNPs, up-conversion nanoparticles; LYM, lymphocytes; BAS, behavioral approach system; MON, monocytes; EOS, eosinophils; NEU, neutrophils; TBIL, total bilirubin level; PLT, platelets; TP, total protein; BUN, blood urea nitrogen; ALT, alanine aminotransferase; CRE, creatinine; PTT, photothermal therapy.

\section{Acknowledgment}

The work was supported by the National Natural Science Foundation of China (81572109).

\section{Author contributions}

All authors contributed to data analysis, drafting and revising the article, gave final approval of the version to be published, and agree to be accountable for all aspects of the work.

\section{Disclosure}

The authors report no conflicts of interest in this work.

\section{References}

1. Joyce JA, Pollard JW. Wmicroenvironmental regulation of metastasis. Nat Rev Cancer. 2009;9(4):239-252.

2. Quail DF, Joyce JA. Microenvironmental regulation of tumor progression and metastasis. Nat Med. 2013;19(11):1423-1437.

3. Mathew R, Karantza-Wadsworth V, White E. Role of autophagy in cancer. Nat Rev Cancer. 2007;7(12):961-967.

4. Folkman J. Angiogenesis and apoptosis. Semin Cancer Biol. 2003;13(2): 159-167.

5. Wang K, Wu X, Wang J, Huang J. Cancer stem cell theory: therapeutic implications for nanomedicine. Int J Nanomedicine. 2013;8:899-908.

6. Dearnaley DP, Khoo VS, Norman AR, et al. Comparison of radiation side-effects of conformal and conventional radiotherapy in prostate cancer: a randomised trial. Lancet. 1999;353(9149):267-272.

7. Lecaros RL, Huang L, Lee TC, Hsu YC. Nanoparticle delivered VEGF-A siRNA enhances photodynamic therapy for head and neck cancer treatment. Mol Ther. 2016;24(1):106-116.
8. Zhang C, Cheng X, Chen M, et al. Fluorescence guided photothermal/ photodynamic ablation of tumours using $\mathrm{pH}$-responsive chlorin e6-conjugated gold nanorods. Colloids Surf B Biointerfaces. 2017;160: 345-354.

9. He Y, Shen Y, Zhou S, et al. Near infrared dye loaded copper sulfideapoferritin for tumor imaging and photothermal therapy. RSC Adv. 2018;8(26):14268-14279.

10. Zhang $\mathrm{C}$, Ren $\mathrm{J}$, Hua J, et al. Multifunctional $\mathrm{Bi}_{2} \mathrm{WO}_{6}$ Nanoparticles for CT-Guided Photothermal and Oxygen-free Photodynamic Therapy. ACS Appl Mater Interfaces. 2018;10(1):1132-1146.

11. Zhao S, Tian Y, Liu W, et al. High and low molecular weight hyaluronic acid-coated gold nanobipyramids for photothermal therapy. RSC $A d v$. 2018;8(16):9023-9030.

12. Sun H, Lv Y, Zhang C, et al. Materials with low dielectric constant and loss and good thermal properties prepared by introducing perfluorononenyl pendant groups onto poly(ether ether ketone). RSC $A d v$. 2018;8(14):7753-7760.

13. Liu Z, Liu J, Wang R, Du Y, Ren J, Qu X. An efficient nano-based theranostic system for multi-modal imaging-guided photothermal sterilization in gastrointestinal tract. Biomaterials. 2015;56:206-218.

14. Yang RM, Fu CP, Fang JZ, et al. Hyaluronan-modified superparamagnetic iron oxide nanoparticles for bimodal breast cancer imaging and photothermal therapy. Int J Nanomedicine. 2017;12:197-206.

15. Thapa RK, Choi JY, Poudel BK, Choi HG, Yong CS, Kim JO. Receptortargeted, drug-loaded, functionalized graphene oxides for chemotherapy and photothermal therapy. Int J Nanomedicine. 2016;11:2799-2813.

16. Zhao P, Ren S, Liu Y, Huang W, Zhang C, He J. PL- $\mathrm{W}_{18} \mathrm{O}_{49}-\mathrm{TPZ}$ Nanoparticles for Simultaneous Hypoxia-Activated Chemotherapy and Photothermal Therapy. ACS Appl Mater Interfaces. 2018;10(4): 3405-3413.

17. Zhang C, Ren J, Yang Y, et al. Ultra-sensitive diagnosis of orthotopic patient derived hepatocellular carcinoma by Fe@graphene nanoparticles in MRI. RSC Adv. 2016;6(115):113919-113923. 
18. Ren S, Cheng X, Chen M, et al. Hypotoxic and Rapidly Metabolic PEGPCL-C3-ICG Nanoparticles for Fluorescence-Guided Photothermal/ Photodynamic Therapy against OSCC. ACS Appl Mater Interfaces. 2017; 9(37):31509-31518.

19. Cantu T, Walsh K, Pattani VP, et al. Conductive polymer-based nanoparticles for laser-mediated photothermal ablation of cancer: synthesis, characterization, and in vitro evaluation. Int J Nanomedicine. 2017; 12:615-632.

20. Wang Z, Chen Z, Liu Z, et al. A multi-stimuli responsive gold nanocagehyaluronic platform for targeted photothermal and chemotherapy. Biomaterials. 2014;35(36):9678-9688.

21. Madani SY, Shabani F, Dwek MV, Seifalian AM. Conjugation of quantum dots on carbon nanotubes for medical diagnosis and treatment. Int J Nanomedicine. 2013;8:941-950.

22. Dong K, Liu Z, Liu J, et al. Biocompatible and high-performance amino acids-capped MnWO4 nanocasting as a novel non-lanthanide contrast agent for X-ray computed tomography and T(1)-weighted magnetic resonance imaging. Nanoscale. 2014;6(4):2211-2217.

23. Liu Z, Liu X, Ran X, Ju E, Ren J, Qu X. Single-layer tungsten oxide as intelligent photo-responsive nanoagents for permanent male sterilization. Biomaterials. 2015;69:56-64.

24. Liu Z, Liu J, Wang R, Du Y, Ren J, Qu X. An efficient nano-based theranostic system for multi-modal imaging-guided photothermal sterilization in gastrointestinal tract. Biomaterials. 2015;56:206-218.
25. Sharifi S, Behzadi S, Laurent S, Forrest ML, Stroeve P, Mahmoudi M. Toxicity of nanomaterials. Chem Soc Rev. 2012;41(6):2323-2343.

26. Nishiyama N, Stapert HR, Zhang GD, et al. Light-harvesting ionic dendrimer porphyrins as new photosensitizers for photodynamic therapy. Bioconjug Chem. 2003;14(1):58-66.

27. Zhang D, Wu M, Zeng Y, et al. Chlorin e6 conjugated poly(dopamine) nanospheres as PDT/PTT dual-modal therapeutic agents for enhanced cancer therapy. ACS Appl Mater Interfaces. 2015;7(15):8176-8187.

28. Xie X, Li Z, Zhang Y, et al. Emerging $\approx 800 \mathrm{~nm}$ excited lanthanide-doped upconversion nanoparticles. Small. 2017;13(6):1602843-1602857.

29. Maeda H, Tsukigawa K, Fang J. A Retrospective 30 years after discovery of the enhanced permeability and retention effect of solid tumors: nextgeneration chemotherapeutics and photodynamic therapy - problems, solutions, and prospects. Microcirculation. 2016;23(3):173-182.

30. Davidson R, Liu Y, Gerien KS, Wu JQ. Real-time visualization and quantification of contractile ring proteins in single living cells. Methods Mol Biol. 2016;1369:9-23.

31. Maeda $H$. Toward a full understanding of the EPR effect in primary and metastatic tumors as well as issues related to its heterogeneity. Adv Drug Deliv Rev. 2015;91:3-6.
International Journal of Nanomedicine

\section{Publish your work in this journal}

The International Journal of Nanomedicine is an international, peerreviewed journal focusing on the application of nanotechnology in diagnostics, therapeutics, and drug delivery systems throughout the biomedical field. This journal is indexed on PubMed Central, MedLine, CAS, SciSearch ${ }^{\circledR}$, Current Contents ${ }^{\circledR} /$ Clinical Medicine,

\section{Dovepress}

Journal Citation Reports/Science Edition, EMBase, Scopus and the Elsevier Bibliographic databases. The manuscript management system is completely online and includes a very quick and fair peer-review system, which is all easy to use. Visit http://www.dovepress.com/ testimonials.php to read real quotes from published authors. 